\title{
Measurement of glomerular filtration rate in patients undergoing obesity surgery
}

Ling L. Chuah', Alexander D. Miras', Laura M. Perry², Andrew H. Frankel ${ }^{3}$, David J. Towey ${ }^{2}$, Zahraa Al-Mayahi ${ }^{*}$, William Svensson ${ }^{4}$ and Carel W. le Roux ${ }^{1,5,6}$

\begin{abstract}
Background: Most studies on obesity surgery have measured renal function using the estimated GFR. However, due to the reduction of muscle mass, and therefore creatinine that accompanies weight loss, such measures can falsely suggest an improvement in renal function. To balance the risks of surgery versus any potential benefits on renal function, we need to be able to determine renal function using valid and reliable methodologies. In this pilot study we aimed to measure renal function in patients with CKD undergoing obesity surgery using the gold standard ${ }^{51} \mathrm{Cr}$-EDTA GFR clearance methodology which is independent of measures of muscle mass.
\end{abstract}

Methods: Nine consecutive obese patients with CKD underwent obesity surgery. Their renal function was assessed using ${ }^{51} \mathrm{Cr}$-EDTA GFR, cystatin $C$ and serum creatinine as well as using eGFR equations including MDRD CKD Epi, Cockcroft Gault and CKD Epi cystatin before and 12 months after surgery.

Results: Renal function using the ${ }^{51} \mathrm{Cr}$-EDTA measured GFR did not change significantly after surgery. Similar results were obtained when Cystatin C, CKD Epi cystatin, CKD Epi cystatin creatinine and adjusted Cockcroft Gault Creatinine clearance methods were used. In contrast there were either trends or significant improvements in renal function measured using the MDRD and CKD Epi equations.

Conclusions: In this pilot study using the gold standard ${ }^{51} \mathrm{Cr}$-EDTA method we found stabilisation in renal function after obesity surgery. Until further definitive data emerge it is critical to balance the risk and benefits of surgery, especially if renal function may not improve as often as previously suggested.

Trial registration: ClinicalTrials.gov NCT01507350. Registered June 2011.

Keywords: Renal, Creatinine, Bariatric, Cr-EDTA, GFR

\section{Background}

Obesity surgery provides significant benefits to obese patients and there is an increasing number of these operations being undertaken in obese patients with chronic kidney disease (CKD) [1]. Previous studies and metanalyses have shown that obesity surgery reduces albuminuria and proteinuria i.e. has a positive effect on renal damage, yet the effect of surgery on renal function is unknown $[2,3]$. The majority of the available literature has measured renal function using the estimated GFR [Modification of Diet in Renal Disease (MDRD)/ chronic

\footnotetext{
* Correspondence: zahraa.al-mayahi14@imperial.ac.uk

1Section of Investigative Medicine, Imperial College London, 6th floor Commonwealth Building, Hammersmith Hospital, Du Cane Road, London W12 0NN, UK

Full list of author information is available at the end of the article
}

kidney disease epidemiology collaboration (CKD Epi)] $[2,4]$. However, due to the reduction of muscle mass, and therefore creatinine, that accompanies weight loss after obesity surgery, such measures can be misleading as numerically calculated eGFR using the MDRD and CKD Epi equations(adjusted to BSA of $1.73 \mathrm{~m}^{2}$ ) may falsely suggest an improvement in renal function [5].

Due to the association between obesity and CKD, an increasing number of patients will qualify for obesity surgery. For patients and clinicians to be able to balance the risks of surgery versus any potential benefits on renal function, we need to be able to determine renal function pre and post operatively using valid and reliable methodologies.

In this pilot study we aimed to measure renal function in patients with CKD 12 months after obesity surgery 
using the gold standard chromium-51 labelled ethylenediamine tetraacetic acid $\left({ }^{51} \mathrm{Cr}\right.$-EDTA GFR $)$ clearance methodology which is independent of measures of muscle mass. We also compared the results from this methodology with those from more commonly used methods.

\section{Methods}

Nine consecutive patients with body mass index (BMI) $\geq 35 \mathrm{~kg} / \mathrm{m}^{2}$ and eGFR (MDRD) $<60 \mathrm{~mL} / \mathrm{min}$ were recruited from our obesity clinic independently of the cause of their CKD. They were eligible for bariatric surgery based on the UK NICE guidelines [6]. Their renal function was assessed using a variety of methods including ${ }^{51} \mathrm{Cr}$-EDTA GFR, cystatin $\mathrm{C}$ and serum creatinine as well as using eGFR equations including MDRD, CKD Epi, Cockcroft Gault and CKD Epi cystatin before and 12 months after bariatric surgery. The body surface area is calculated according to the DuBois formula [7].

${ }^{51} \mathrm{Cr}$-EDTA GFR was ascertained using bolus injection of $1.46-2.66 \mathrm{MBq}{ }^{51} \mathrm{Cr}$-EDTA, with between 6 and 8 venous blood samples were collected at approximately $15,30,60,90,120,180,240$, and $300 \mathrm{~min}$ to assess ${ }^{51} \mathrm{Cr}$-EDTA clearance. ${ }^{51} \mathrm{Cr}$-EDTA GFR was calculated using the Bi-exponential Fitting Method described in the British Nuclear Medicine Society guidelines [8]. The prepared standard and patient samples were counted for 15 and 60 mins respectively using a Wallac 1470 Wizard Gamma Counter (Perkin Elmer Inc., Waltham, Massachusetts, USA). The measured GFR was scaled to BSA in order to maintain uniformity in comparison to reported eGFR.

Descriptive statistics were expressed as median (interquartile range) given the sample size of 9 subjects. Within groups, comparisons were made using the Wilcoxon matched pairs test given the sample size. The Pearson methodology was used to test for correlations (Graphpad PRISM software version 5.01). Statistical significance was accepted as $p<0.05$. This was a pilot study without formal a priori power calculations.

\section{Results}

Subject characteristics are shown in Table 1. Subjects had diabetic kidney disease $(n=1)$, non-steroidal antiinflammatory drug induced nephropathy $(n=1)$, unilateral nephrectomy for renal cell carcinoma $(n=1)$, hypertensive nephropathy $(n=1)$, and unknown causes of CKD $(n=3)$. Two subjects had renal transplantation before obesity surgery secondary to diabetic kidney disease and focal segmental glomerulosclerosis respectively. Seven subjects had Roux-en-Y gastric bypass (RYGB), one had adjustable gastric banding, and one had a vertical sleeve gastrectomy (VSG).
Table 1 Pre and post-operative measurements

\begin{tabular}{|c|c|c|c|}
\hline & Pre-op $(n=9)$ & Post-op $(n=9)$ & $P$ value \\
\hline Age (year) & $62.0(50.5-63.0)$ & & \\
\hline Male $(\%, n)$ & $55.56 \%, 5$ & & \\
\hline Weight (kg) & $127.0(116.5-144.8)$ & $100.2(83.6-110.9)$ & 0.004 \\
\hline BMI $\left(\mathrm{kg} / \mathrm{m}^{2}\right)$ & $45.5(42.2-47.8)$ & $34.3(29.3-38.3)$ & 0.008 \\
\hline $\operatorname{BSA}\left(m^{2}\right)$ & $2.28(2.18-2.45)$ & $2.08(1.97-2.14)$ & 0.004 \\
\hline Creatinine $(\mu \mathrm{mol} / \mathrm{L})$ & $122.5(96.8-149.5)$ & $110.0(88.0-116.0)$ & 0.01 \\
\hline Cystatin C(mg/L) & $1.6(1.3-2.1)$ & $1.7(1.4-1.8)$ & 0.64 \\
\hline EDTA GFR & $58.0(48.5-91.0)$ & $59.0(48.9-72.5)$ & 0.38 \\
\hline $\begin{array}{l}\text { EDTA GFR corrected for } \\
\text { BSA }\left(\mathrm{ml} / \mathrm{min} / 1.73 \mathrm{~m}^{2}\right)\end{array}$ & $43.6(37.0-65.0)$ & $45.8(41.7-63.2)$ & 0.30 \\
\hline $\begin{array}{l}\text { MDRD eGFR } \\
\left(\mathrm{mL} / \mathrm{min} / 1.73 \mathrm{~m}^{2}\right)\end{array}$ & $51.0(38.3-60.3)$ & $53.5(42.3-65.3)$ & 0.08 \\
\hline CKD Epi $\left(\mathrm{mL} / \mathrm{min} / 1.73 \mathrm{~m}^{2}\right)$ & $54.0(42.0-65.0)$ & $61.0(48.5-71.0)$ & 0.008 \\
\hline $\begin{array}{l}\text { CKD Epi cystatin } \\
\left(\mathrm{mL} / \mathrm{min} / 1.73 \mathrm{~m}^{2}\right)\end{array}$ & $39.0(27.0-49.0)$ & $40.0(34.5-49.8)$ & 1.0 \\
\hline $\begin{array}{l}\text { CKD Epi cystatin } \\
\text { creatinine } \\
\left(\mathrm{mL} / \mathrm{min} / 1.73 \mathrm{~m}^{2}\right)\end{array}$ & $43.0(33.0-56.0)$ & $47.5(42.0-54.8)$ & 0.34 \\
\hline $\begin{array}{l}\text { Cockcroft Gauld } \\
\text { Creatinine clearance, } \\
\left.\text { adjusted (mL/min/1.73 } \mathrm{m}^{2}\right)\end{array}$ & $77.2(61.6-89.0)$ & $75.0(64.1-92.3)$ & 0.93 \\
\hline
\end{tabular}

Abbreviations: $B M I$ body mass index, BSA body surface area, CKD Epi chronic kidney disease epidemiology collaboration, EDTA ethylenediamine tetraacetic acid, eGFR estimated glomerular filtration rate, MDRD modification of diet in renal disease

After surgery BMI and creatinine reduced significantly. There were no major complications peri-operatively for any of the patients and none developed symptomatic kidney stones. There was no significant change in the number of blood pressure lowering medications [2.0 (1.3-2.0) vs. $2.0(0.5-2.0), p=1.0]$.

Renal function using the ${ }^{51} \mathrm{Cr}$-EDTA measured GFR did not change significantly after surgery (Fig. 1).

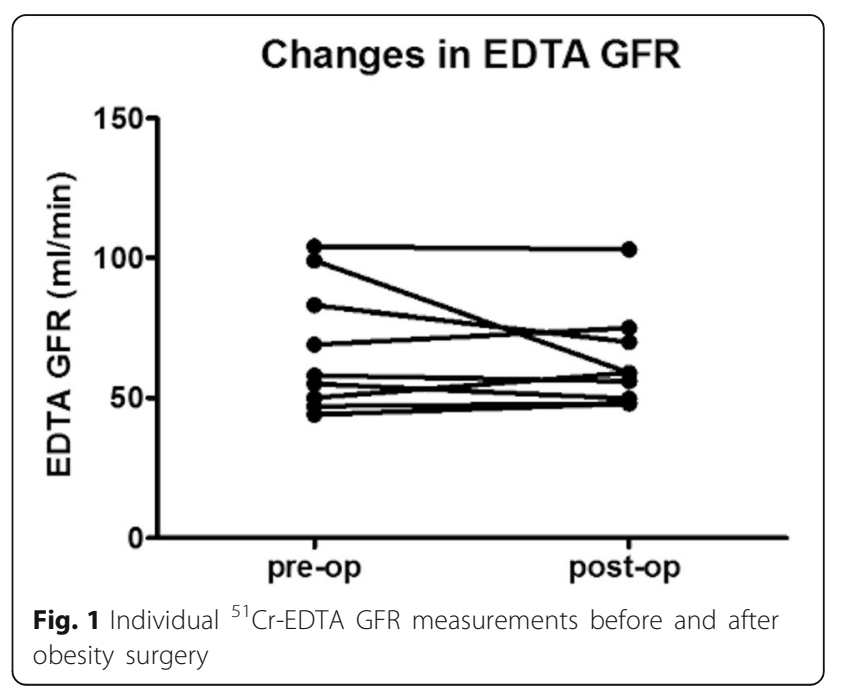


Similar results were obtained when Cystatin C, CKD Epi cystatin, CKD Epi cystatin creatinine and adjusted Cockcroft Gault Creatinine clearance methods were used (Table 1). In contrast there were either trends or significant improvements in renal function measured using the MDRD and CKD Epi equations. When the unadjusted Cockcroft Gault Creatinine clearance methodology was used, there was a reduction in renal function as the equation incorporates weight into the measure.

\section{Discussion}

This pilot study has demonstrated that renal function as measured by the gold standard ${ }^{51} \mathrm{Cr}$-EDTA GFR method remained stable 12 months after bariatric surgery. This is in contrast to trends for or significant improvements in renal function when assessed using the MDRD equation or CKD Epi. MDRD GFR and CKD epi are known to underestimate mGFR as these methodologies are dependent on the serum creatinine, which is in turn influenced by total muscle mass. Following surgery, the reduction in weight and serum creatinine led to increase in MDRD GFR and CKD Epi, bringing it closer to mGFR.

We presented mGFR and mGFR corrected to BSA. Whilst recognised that indexation of glomerular filtration rate for BSA in obese patients could underestimate the real GFR [9], due to the reduction in significant amount of weight in obese patient post weight loss surgery; we reported the mGFR correction for BSA in order to facilitate comparison with reported eGFR corrected for BSA.

The current literature on the effects of obesity surgery on renal outcomes appears promising [4]. Surgery has been reported to reduce glomerular hyperfiltration, albuminuria and proteinuria in obese patients with or without type 2 diabetes mellitus [2]. In well matched observational cohort studies obesity surgery was associated with a $58 \%$ lower risk of eGFR decline of $\geq 30$ and $57 \%$ lower risk of serum creatinine decline or development of end-stage renal disease in patients with and without CKD [10]. However, these findings need to be interpreted with caution as GFR was estimated and not actually measured. Within our small study group, stabilisation of renal function was found; which is a positive and encouraging finding is in itself.

Reassuringly our findings are consistent with another study that assessed 12 patients with CKD undergoing RYGB, VSG and gastric banding using BSA unadjusted iothalamate measured GFR and which also found stabilisation of measured GFR [11]. Interestingly, in the same study, measured GFR increased when adjustment for BSA was made. Similar findings have been observed when GFR was measured in patients with preserved renal function undergoing obesity surgery [4].
In our study stabilisation in renal function was also demonstrated using methodologies that incorporate direct measurement of cystatin C, such as CKD Epi cystatin creatinine. Cystatin $C$ levels are not related to body or muscle mass and because EDTA GFR can be cumbersome and resource demanding, measurement of Cystatin $\mathrm{C}$ may be a useful alternative from a practical perspective.

Our study was limited by the small sample size, the heterogeneity of the aetiology of renal disease at baseline and type of operation used. Definitive interrogation of the effects of obesity surgery on measured GFR needs to be assessed in a prospective study with less aetiological heterogeneity, on type of operation used and longer follow up, as it can take years before any changes of GFR become evident.

\section{Conclusions}

In conclusion we found stabilisation in renal function as measured by ${ }^{51} \mathrm{Cr}$-EDTA GFR or Cystatin $\mathrm{C}$ related assessments in patients with CKD 12 months after obesity surgery. Until further data emerges, where the surgical risk is high, it is critical to balance the risk and benefits of obesity surgery, especially if renal function may not improve as often as previously suggested.

\section{Abbreviations \\ BMI: Body mass index; CKD Epi: Chronic kidney disease epidemiology collaboration; EDTA: Ethylenediamine tetraacetic acid; eGFR: Estimated glomerular filtration rate; MDRD: Modification of diet in renal disease}

\section{Acknowledgements}

The authors thanked Dr. Wiebke Fenske for helping obtain ethics approval for the study and the multidisciplinary obesity team for the care provided to our patients.

\section{Funding}

The study was funded by the J P Moulton Charitable Foundation. The funding body did not have any role in the design of the study and collection, analysis, and interpretation of data and in writing the manuscript. ADM has received funding from an MRC Clinical Training Fellowship, MRC Centenary Award, and an NIHR Clinical Lectureship. CWIR is funded by Science Foundation Ireland /YI/B2480

\section{Availability of data and materials}

The datasets used and/or analysed during the current study are available from the corresponding author on reasonable request.

\section{Authors' contributions}

Conduct of the experiments: LLC, ADM; laboratory analyses: LMP, DJT, WS, ZAM; data analyses: LLC, ADM, ZAM; manuscript write up: all authors; study concept: AHF, CWIR; guarantor of data accuracy: CWIR. All authors have read and approved the final version of this manuscript.

\section{Ethics approval and consent to participate}

The study was approved by the local research and ethics committee (West London Research Ethics Committee, Reference 10/H0707/96). Patients provided written informed consent and the study was conducted according to the principles of the Helsinki declaration.

Consent for publication

Not applicable.

Competing interests

The authors declare that they have no competing interests. 


\section{Publisher's Note}

Springer Nature remains neutral with regard to jurisdictional claims in published maps and institutional affiliations.

\section{Author details}

'Section of Investigative Medicine, Imperial College London, 6th floor Commonwealth Building, Hammersmith Hospital, Du Cane Road, London W12 ONN, UK. ${ }^{2}$ Radiological Sciences Unit, Imperial College Healthcare NHS Trust, London, UK. ${ }^{3}$ Nephrology, Imperial College Healthcare NHS Trust, London, UK. ${ }^{4}$ Nuclear Medicine, Imperial College Healthcare NHS Trust, London, UK. ${ }^{5}$ Gastro Surgical laboratory, University of Gothenburg, Gothenburg, Sweden. ${ }^{6}$ Diabetes Complications Research Centre, Conway Institute, University College Dublin, Dublin, Ireland.

Received: 12 July 2018 Accepted: 17 December 2018

Published online: 29 December 2018

\section{References}

1. Friedman AN, Wolfe BI. Bariatric Surgery an effective treatment for type ॥ diabetic kidney disease? Clin J Am Soc Nephrol. 2016;11(3):528-35.

2. Li K, Zou J, Ye Z, et al. Effects of Bariatric Surgery on renal Function in obese patients: a systematic review and Meta analysis. PLoS One. 2016;11(10): e0163907.

3. Miras AD, Chuah LL, Khalil N, et al. Type 2 diabetes mellitus and microvascular complications 1 year after roux-en-Y gastric bypass: a case-control study. Diabetologia. 2015;58(7):1443-7.

4. Chang AR, Grams ME, Navaneethan SD. Bariatric Surgery and Kidney-Related Outcomes. Kidney Int Rep. 2017;2(2):261-70. https://doi.org/10.1016/j.ekir. 2017.01.010 Epub 2017 Jan 1026

5. Levey AS, Bosch JP, Lewis JB, Greene T, Rogers N, Roth D. A more accurate method to estimate glomerular filtration rate from serum creatinine: a new prediction equation. Modification of diet in renal disease study group. Ann Intern Med. 1999:130(6):461-70.

6. Excellence NifhaC. Obesity: identification, assessment and management. 2014. www.nice.org.uk/quidance/cg189/chapter/1-recommendations.

7. Du Bois D, Du Bois EF. A formula to estimate the approximate surface area if height and weight be known. 1916. Nutrition 1989;5(5):303-311; discussion 312-303.

8. Fleming JS, Zivanovic MA, Blake GM, Burniston M, Cosgriff PS. Guidelines for the measurement of glomerular filtration rate using plasma sampling. Nucl Med Commun. 2004;25(8):759-69.

9. Delanaye P, Cohen EP. Formula-based estimates of the GFR: equations variable and uncertain. Nephron Clin Pract 2008;110(1):c48-c53; discussion c54 doi: https:/doi.org/10.1159/000151436. Epub 000152008 Aug 000151418.

10. Chang AR, Chen Y, Still C, et al. Bariatric surgery is associated with improvement in kidney outcomes. Kidney Int. 2016;90(1):164-71.

11. Navaneethan SD, Malin SK, Arrigain S, Kashyap SR, Kirwan John P, Schauer PR. Bariatric Surgery, Kidney Function, Insulin Resistance, and Adipokines in patients with decreased GFR: a cohort study. Am J Kidney Dis. 2015;65(2): $345-7$.

Ready to submit your research? Choose BMC and benefit from:

- fast, convenient online submission

- thorough peer review by experienced researchers in your field

- rapid publication on acceptance

- support for research data, including large and complex data types

- gold Open Access which fosters wider collaboration and increased citations

- maximum visibility for your research: over $100 \mathrm{M}$ website views per year

At $\mathrm{BMC}$, research is always in progress.

Learn more biomedcentral.com/submissions 